\title{
Towards the timely detection of toxicants
}

\author{
Massimiliano Ignaccolo ${ }^{1, *}$, Paolo Grigolini ${ }^{1,2,3}$, and Guenter Gross ${ }^{4}$ \\ ${ }^{1}$ Center for Nonlinear Science, University of North Texas, \\ P.O. Box 311427, Denton, Texas 76203-1427 \\ ${ }^{2}$ Dipartimento di Fisica dell'Università di Pisa and INFM, Via Buonarroti 2, 56127 Pisa, Italy \\ ${ }^{3}$ Istituto di Biofisica del Consiglio Nazionale delle Ricerche, \\ Area della Ricerca di Pisa, \\ Via G. Moruzzi 1, 56124, Italy and \\ 4 University of North Texas Center for Network Neuroscience
}

(Dated: October 30, 2018)

\begin{abstract}
We address the problem of enhancing the sensitivity of biosensors to the influence of toxicants, with an entropy method of analysis, denoted as CASSANDRA, recently invented for the specific purpose of studying non-stationary time series. We study the specific case where the toxicant is tetrodotoxin. This is a very poisonous substance that yields an abrupt drop of the rate of spike production at $\mathrm{t}$ approximatively 170 minutes when the concentration of toxicant is 4 nanomoles. The CASSANDRA algorithm reveals the influence of toxicants thirty minutes prior to the drop in rate at a concentration of toxicant equal to 2 nanomoles. We argue that the success of this method of analysis rests on the adoption of a new perspective of complexity, interpreted as a condition intermediate between the dynamic and the thermodynamic state.
\end{abstract}

\section{INTRODUCTION}

One of the issues raised by September 11 is the timely detection of toxicants. Cell-based biosensors have proven to afford an efficient way to monitor the presence in the environment of toxicants, pathogens and other agents 1 . In fact, upon increase of time the function of the neural network (biosensor) is significantly affected by the action of the toxicant, and the experimental results show that the firing process undergoes significant changes under the presence of toxicants. This important property of biosensors is shown by the example of Fig. 1 . At time $t_{1}=30$ minutes a given initial concentration of toxicant, tetrodotoxin molecule, in this case, is added to the compound, and it is steadily increased in time till at time $t_{2} \approx 170$ minutes, where by visual inspection we see an abrupt change in the time dependence of the spike rate for minutes, namely, the total number of spikes detected in one minute, divided by the number of neurons monitored. A further increase in the concentration of tetrodotoxin fully inihibits the spike production at $t_{3} \approx 400$ minutes.

A theoretical challenge, for the Science of Complexity, is to detect the presence of toxicant much earlier than the abrupt change in the firing rate. This might become a question of death or life, if the concentration of toxicants producing the abrupt change is very close to the lethal concentration. An interesting issue is the following: Let us consider the early portion of Fig. 1 , in the time region preceding, let us say, $t_{4} \approx 150$ minutes. Are there slight dynamical changes that an ordinary visual inspection does not detect, while a careful statistical analysis done with a fast computational algorithm can? This is a problem of significant practical importance, but at the same time is a theoretical challenge. In fact, if there are slight changes, their intensity is expected to increase with time. This means that the time series is not stationary, the statistical analysis of a non-stationary time series being perceived as a challenge. The title of the October 2002 Workshop, whose Proceedings are published in this issue of Chaos, Solitons and Fractals, containing this as well as many other papers originally presented in this Workshop, is: Non-stationary Time Series: A Theoretical, Computational and Practical Challenge. The main purpose of this paper is to address this challenging issue, in the special case of biosensors, whose function is altered in time to an extent that it is not easy to detect via mere visual inspection. We shall prove that it is possible to address this issue with success, and that the results obtained can be used to improve the biosensor technique, with a significant progress towards the main goal of making a timely detection of toxicants.

\section{THE CASSANDRA METHOD}

The name CASSANDRA is the acronym for Complex Analysis of Sequences via Scaling AND Randomness Assessment 2]. The authors of Ref. [2] proposed this algorithm for the specific purpose of studying non-stationary time

\footnotetext{
* Corrispondent Author. Email: mi0009@unt.edu (active till the end of September) or m_ignaccolo@yahoo.it
} 


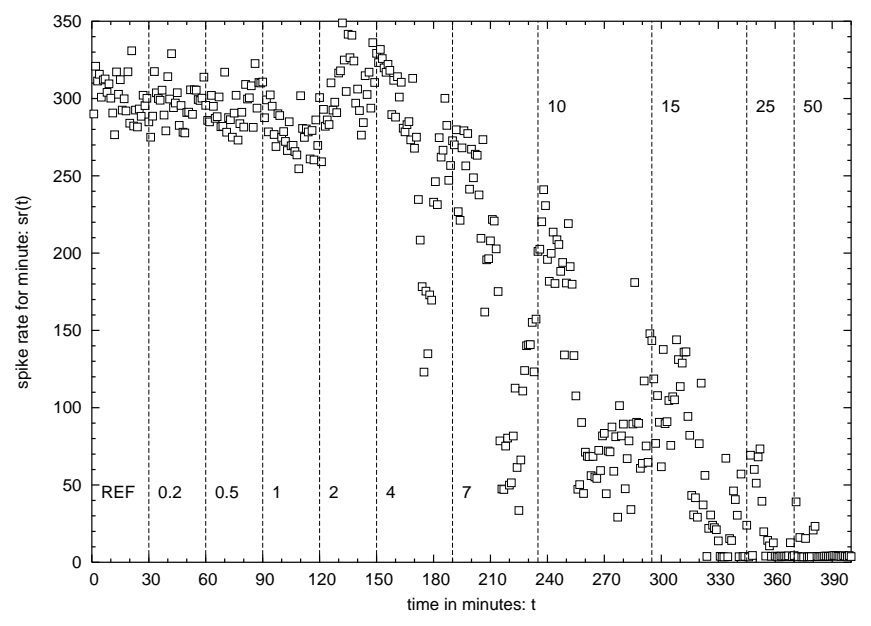

FIG. 1: The average spike rate for minute for the monitored potion of the network: 67 neurons. The vertical lines signal the borders of states with a given concentration of tetradotoxin. The numbers between two consecutive vertical lines denote the concentration of tetrodotoxin in nano moles, characterizing the corresponding state. The label REF indicates the network state chosen as the reference for this experiment. In our case the reference state is 30 minutes of native (no toxicant added) activity where the average spike rate for minute is fairly stable.

series. It is worth reviewing the research work that led the authors of Ref. [2] to the foundation of this method. This is important because, as we shall see, this critical review has the effect of proving that CASSANDRA is the natural consequence of defining complexity as a condition intermediate between the dynamic and the thermodynamic state. This view of complexity, denoted as Living State of Matter (LSM), has been advocated by the authors of the work of Ref. 3], proving the fruitful use of this perspective for the foundation of a new theory for absorption and emission of light in nano-materials. Actually, the authors of Ref. [3] inspired their approach to non-living materials to the perspective proposed by two professional biologists, whose ideas are reviewed in a paper of these Proceedings [4]. We hope that the efficiency itself of the CASSANDRA algorithm, confirmed by the results of the present paper, might afford further support to the convenience of adopting the LSM perspective.

In principle, an attractive way to establish whether a time series is totally random or not is given by the KolmogorovSinai (KS) entropy [5]. However, as widely discussed in an earlier publication [6], there are problems with the computation estimate of the KS entropy. Therefore, the authors of Ref. [6] recommended the recourse to two calculation techniques that are apparently different but actually closely related the one to the other. The first technique is the compression technique that rests on establishing if a time series of a given length can be replaced by a much shorter sequence, yielding the same information. From an intuitive point of view, this compression is possible when the time series under study is characterized to some extent by regularity. The second technique discussed in Ref. [6] is a method introduced for the first time by the authors of Ref. [7] and Ref. [8]. This method of analysis is based on converting the time series under study into a diffusion process. This is done as follows. Let us denote by $\left\{\xi_{i}\right\}$ the time series under study, with a total length $N$ that we assume to be very large. We define a discrete time $l$, which is the size of a window that we move along the sequence. For any window position $s, s=1,2, \ldots, N-l+1$, we select the values of the sequence spanned by the window and we create the $s$-th diffusion trajectory defined by

$$
x^{(s)}=\sum_{i}^{l} \xi(s+i) .
$$

We consider this as the $s$-th random walker of a set of $N-l+1$ random walkers, moving for a time $l$, from the position $x=0$ at time $l=0$. If the time series under study is totally random, the random walkers are not correlated, and the resulting diffusion process will be very close to the condition of ordinary diffusion. The method of Ref. [] and Ref. 8] rests on evaluating the Shannon entropy of the resulting diffusion process. For this reason the method was called Diffusion Entropy (DE) method. According to a popular tenet of the Science of Complexity [9], complexity is related to the concept of scaling. In the case of a diffusion process, with diffusion distribution density $p(x, l)$, scaling is defined by

$$
p(x, l)=\frac{1}{l^{\delta}} F\left(\frac{x}{l^{\delta}}\right) .
$$

Complex systems are expected to generate a departure from the condition of ordinary diffusion, where $\delta=0.5$ and 
$F(y)$ is a Gaussian function of $y$. This is very attractive and the DE method yields the correct scaling coefficient even when the function $p(x, l)$ does not have a finite second moment, thereby invalidating the method of scaling detection based on the variance evaluation. The DE method, in the case when the property of Eq. (2) applies, it is easily proved to yield

$$
S(l)=A+\delta \ln (l),
$$

where $A$ is a constant whose explicit expression is of no interest here. It is evident that with this method the scaling parameter is easily evaluated by plotting $S(l)$ in a linear-log representation.

However, in the case of a complex system, characterized by anomalous scaling $\delta$, the time necessary for the system to make a transition to the scaling regime, is extremely large and ideally infinite, as proved by the authors of Ref. [10]. The work of Ref. 10] suggests a very attractive way to define complexity. Complexity is not so much represented by an anomalous scaling, but rather by the transition regime necessary to reach the scaling regime. The scaling regime is equivalent to the condition of thermodynamic equilibrium. This is so because the scaling condition, as the standard canonical equilibrium, is eventually realized moving from any non-equilibrium (non-scaling) condition. However, it takes an infinite, rather than a finite time, to realize the condition of anomalous scaling. This would not be a problem, if the concentration of tetrotodotoxin were kept constant. In practice, we guess that in a suitably large time scale, of the order days, for instance, one would be able to detect the anomalous scaling of the compound. The scaling condition, in other words, is realized at a so large time scale, where the memory effects produced by cooperation are lost, and the LSM properties have been annihilated. In this scaling condition the deviation of $\delta$ from 0.5 and the deviation of $F$ from the Gaussian shape, signal that something unusual occurred, when the system was alive, namely, during the transition from the dynamic to the thermodynamic condition. The anomalous property is the long time spent by the system in a condition intermediate between dynamics and thermodynamics. It is evident, therefore, that measuring the scaling in practice is impossible when the dynamic rules are changed in a time scale of the order of minutes. On the other hand, the anomalous scaling is a signature of an anomalous transient condition, of significant conceptual interest, which is even more important than the anomalous scaling itself.

For all these reasons, the authors of Ref. 2] invented the CASSANDRA algorithm. The name of this statistical method of analysis seems to be very appropriate for the purpose of this paper, since we shall be using it to predict much earlier the occurrence of the toxicant-induced abrupt change of the firing activity. This is a non-wished event belonging to the category of those that the prophetess Cassandra would have predicted, and nobody would have believed her. We shall prove that the CASSANDRA algorithm is as accurate as the predictions of Cassandra prophetess, and we shall prove that it deserves the reader's trust. The CASSANDRA method works as follows. As pointed in Section 1, we study a time series that is not stationary. This means that the rules change with time. However, let us make the assumption that the change is slow enough that a portion of the time series of length $L$ can be regarded as being a stationary sequence. Then, we supplement the DE method with the introduction of a big window of size $L$, which has to be considered as a sequence of its own, and we move it along the sequence to study, for the main purpose of assessing its local properties. The size of this window has to be large enough as to make it possible to make a statistical analysis. This means that for any window position $j$ we must span a significant portion of the sequence $\{\xi\}$ under study, so as to establish genuinely local properties. For each position of the big window, using the procedure earlier described to create diffusion, with small windows of size $l$, we evaluate the quantity

$$
C_{j}(\lambda)=\frac{\sum_{l=1}^{\lambda}\left[S_{j}(l)-S_{j}(1)-0.5 \ln (l)\right]}{\lambda},
$$

where $S_{j}(l)$ and $S_{j}(1)$ denote the Shannon entropies of the diffusion processes corresponding to small windows of size $l$ and 1 , respectively, moving within the big window with position $j$. The meaning of the prescriptionof Eq. (4) is transparent. We compare the actual entropy change to the ideal change occurring with an infinitely fast transition from dynamics to thermodynamics, this corresponding, in fact, in the case of ordinary diffusion, to the diffusion entropy increasing as $0.5 \ln (l)$. Note that the sequence $\{\xi\}$ will be built up in Section 4 with a prescription that is the specific way we use to process the data of Fig. 1. This prescription will establish a correspondence between the index $j$ and the continuous time $t$, so that the index $j$ can be identified with the continuous time $t$. Thus, for any value of the sequence $\{\xi\}$ we know the concentration of toxicant, which increases as we move from the smaller to the larger values of the index $j$, denoting the sequence site.

In conclusion, the validity of Eq. (44) as a measure of the local deviation from the diffusion entropy increase that would be generated by random fluctuations, rests on the mathematical inequality

$$
\lambda<<<<N .
$$

This is so because with the condition $L<<N$, we can locate the big window in different positions of the sequence $\{\xi\}$, corresponding to no toxicants, to a small concentration of toxicants or to a large concentration of toxicants, as we wish. 
The condition $\lambda<<L$ makes it possible for us to use enough data so as to reach a conclusion about the statistical property of the small region under observation. We see from Eq. (4) that $C_{j}(\lambda)$ affords a significant information about the local process of transition from dynamics to thermodynamics. If the small region under observation is totally random, the transition to the scaling regime, which in this case would be ordinary, is very quick, and the CASSANDRA indicator yields a virtually vanishing value [11]. If, on the contrary, the small region under observation is compressible, in the sense of Kolmogorov, as discussed in Section 2, the transition from dynamics to thermodynamics (scaling regime) becomes much slower, and the prescription of Eq. (4) yields values with a significantly larger modulus, which, moreover, change with the changes of the rules driving the dynamics of the process. This is the reason of the sensitivity to a changing toxicant concentration, which is, in fact, expected to change the dynamic rules of the process.

\section{TIMELY DETECTION OF TOXICANTS}

This section is devoted to illustrating our method of analysis in action on the analysis of the spike production of biosensors under the influence of tetrodotoxin. Tetrodotoxin is one of the most potent molecules known. Once introduced, it selectively blocks the voltage-sensitive sodium channels of excitable tissues. As a result, tetrodotoxin inhibits or reduces the chances of an action potential to be produced. Tetrodotoxin is complex in structure and contains a imidazole ring. It is likely that this ring is the part of the molecule that lodges in the channel leaving the rest of the molecule blocking its outer mouth. It is therefore of crucial importance to enhance the sensitivity of biosensors to this poisoning molecule. In the first part of this section we describe how the experimental data are processed, regardeless of whether this toxicant is acting or not. Now we shall discuss this method of analysis in action with the concentration of tetrodotoxin increasing in time.

\section{A. Random Walk representation of the neural netwotk activity}

The neural network (biosensor) is a composed by many neurons, but it is possible to monitor only the activity of a part of it 1]. For each neuron whose activity can be monitored we have at our disposal, the time series representing the time at which the neuron fires (time stamp series). Using the information about the single neuron, we can build up a time series representing the temporal evolution of the activity of the monitored neurons. This is done dividing the time domain into intervals of time (bins) of duration $\Delta$ (bin size) bigger or equal to the time resolution of the time stamp series and counting the number spikes registered during each interval of time. Fig. 2 shows how 1 minute of activity of the monitored neurons is reproduced with the binning procedure described above $(\Delta=1 / 100$ seconds $)$.

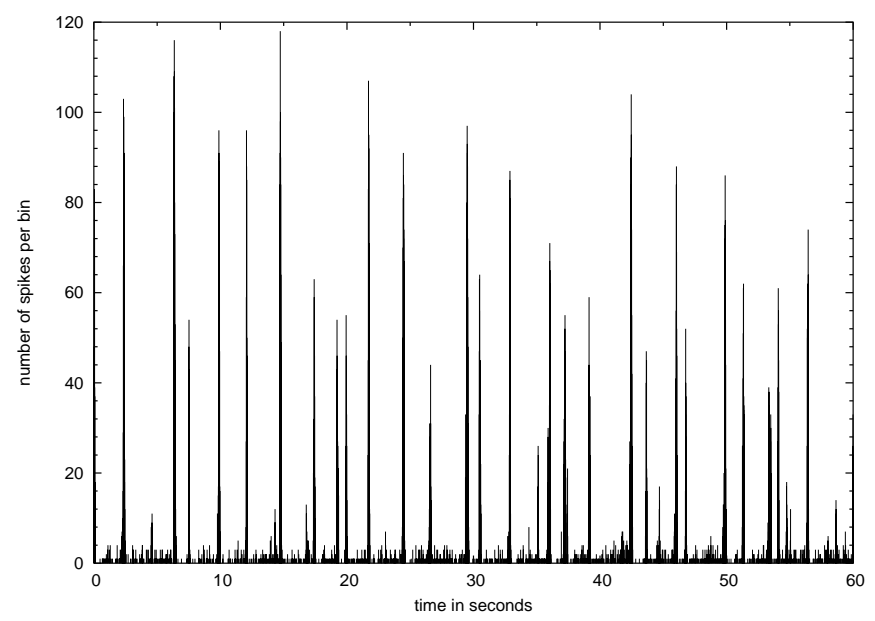

FIG. 2: A sample of our method of data processing. This is the number of spikes per bin, during the first minute of activity of the neural network. During this period of time no tetrodotoxin is present in the neural network.

The reason for this special way of data processing is to create conditions corresponding to the complexity perspective established by the work of Refs. [2, 6, 8], whose fundamental prescription is in fact to turn the time series to analyze into a continuous time random walk, and, this, in turn, into a diffusion process. For any value of time of the abscissa axis, the random walker makes a jump ahead by a quantity proportional to the number of spikes per bin. This is a 
realization of the prescriptions of Ref. [8]. Since we do not aim at establishing the scaling, as in Ref. [8] for the reasons widely detailed in Section 1, we do not care about the statistics of the jump intensities. In fact, we shall be using CASSANDRA, and this algorithm will focus only on how much the onset of resulting diffusion process departs from the ideally instantaneous transition to ordinary diffusion.

\section{B. Experiment and Results}

As explained in Section 1 by the illustration of Fig. 1, the experiment rests on adding the tetrodotoxin molecule to the compound, with a concentration increasing with time. To make easier for the reader to appreciate the benefit of using CASSANDRA, in Fig. 3 we illustrate again the experimental results of Fig. 1, with a first significant change occurring at about 170 minutes, the location of the first dip. Note that Fig. 3 refers to the case where the bin size has the value $\Delta=0.01 s$. We see that CASSANDRA produces its first significant dip at 135 minutes, namely 35 minutes earlier! Changing the bin size from $0.001 \mathrm{~s}$ to $0.1 \mathrm{~s}$ does not change the result. This means that the changes observed by CASSANDRA are produced by internal dynamics corresponding to that time scale.

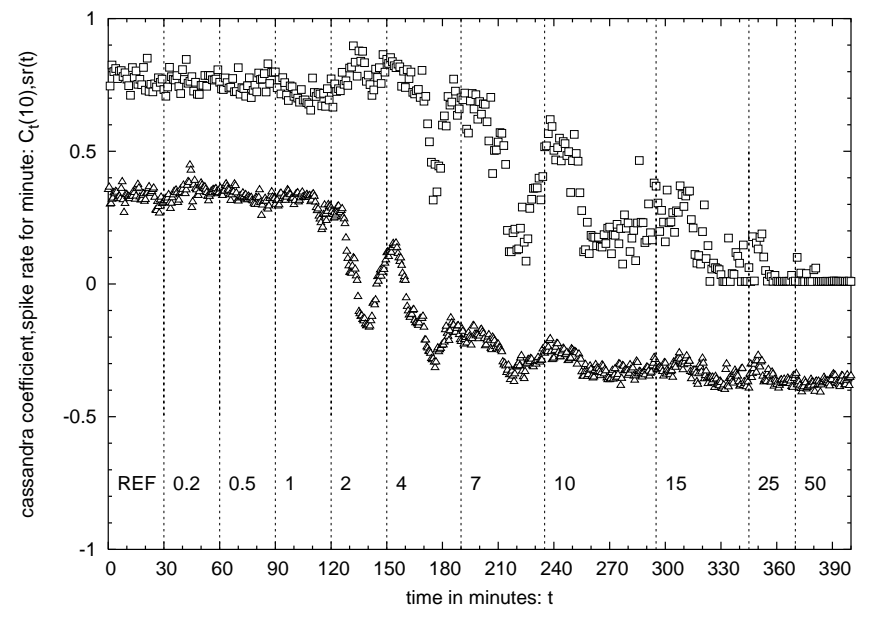

FIG. 3: The CASSANDRA coefficient (triangles) $C_{t}$ for $\lambda=10$, as a function of time, compared to a properly rescaled average spike rate for minute (squares). Note that the index $j$ of the prescription of Eq. (4) is replaced by the corresponding time $t$, which can be thought of as continuous. The meaning of the vertical lines is the sams as that of Fig. 1.
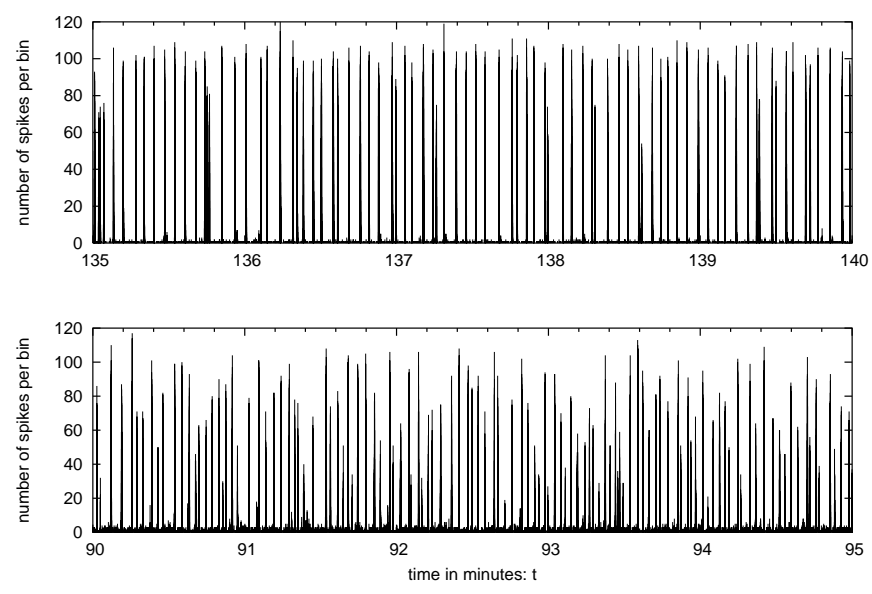

FIG. 4: Comparison of two different neural network activities. The bottom frame refers to the time region betwen 90 and 95 minutes with the concentration of 1 nanomole. The top frame refers to the time region between 135 and 140 minutes, with a concentration of 2 nanomoles.

The importance of this result within the scenario of the bio-terrorism threat is evident. However, here we want to focus our attention on the use of CASSANDRA as a way to deepen our understanding of biosensor dynamics. To shed 
more light into this issue, let us make some comments regarding Fig. 4. This figure illustrates the spike production occurring between 135 and 140 minutes and between 90 and 95 minutes. In the latter time interval the concentration is one nano-mole and, at this small concentration, not even the CASSANDRA algorithm succeeds in signaling the toxicant presence. In the former, where the toxicant concentration is of 2 nano-moles, on the contrary, CASSANDRA yields its first dip, thereby signaling the toxicant presence, while the spike rate for minute remains fairly stable (see Fig. 3). The difference between the two kinds of activity is made evident by these two frames. In the top frame the collective burst events (high value of the number of spikes per bin) are more regular in intensity (number of spikes) and, on average, keep one from the next a time distance bigger than in the bottom frame. In other words, there is a trade between time distance and intensity that has the effect of leaving almost unchanged the rate of spike production. These two different ways of realizing the same rate of spikes per minutes is not overlooked by CASSANDRA, which in fact signals the difference between the two conditions with the significant dip at $t \approx 135$ minutes.

\section{CONCLUSION}

We are convinced that the surprising efficiency of CASSANDRA can be given a convincing explanation, based on the LSM perspective [3, 4]. In fact, the way we used to process the data enhances the cooperative effect of the biosensors. These cooperative effects are the main reason why the transition to scaling is delayed with respect to ordinary Brownian motion. This is also the reason why, as pointed out in Section 1, the adoption of a scaling detector would not afford any significant information. In fact, the attainment of the scaling condition is very slow, much slower than the change of toxicant concentration as a function of time. The enhancement of the collective properties corresponds to moving from the detailed observation of a single channel, which might give the misleading impression of dealing with dynamics, to the observation of collective properties, related to the main idea of biosensors as an orchestra, where the individual players are coordinated by the orchestra director. Our LSM perspective refers to this cooperative condition, which we do not have to confuse with the dynamic condition, taking place at a much shorter time scale. The time scale between $0.1 \mathrm{~s}$ and $0.001 \mathrm{~s}$ is where the cooperative effects are significant. This is why the transition to the scaling condition is very slow, with details that are sensitive to the influence of toxicants. The toxicant influences the cooperative behavior and consequently the regime of transition to scaling (the thermodynamic regime). The CASSANDRA algorithm, focusing on the observation of how the entropy increase departs from the ideal behavior of a diffusion process with an instantaneous transition to scaling, makes it possible to detect these changes. The importance of this result within the threatening scenario of bio-terrorism is evident.

P.G. and M.I. acknowledge support from ARO, through Grant DAAD19-02-0037.

[1] D. A. Stenger, G.W. Gross, E. W. Keefer, K. M. Shaffer, J. D. Andreadis, W.Ma and J. J. Pancrazio, Detection of physiologically active compunds using cell-based biosensors, TRENDS in Biotechnology, 19, 304 (2001).

[2] P. Allegrini, P. Grigolini, P. Hamilton, L. Palatella, G. Raffaelli, M. Virgilio, in Emergent Nature, ed. by M.M. Novak, World Scientific, Singapore (2001) p. 183.

[3] P. Allegrini, G. Aquino, P. Grigolini, L. Palatella, A. Rosa, cond-mat/0304506

[4] M. Buiatti, M. Buiatti, Towards a characaterization of the living state of matter, Chaos, Solitons and Fractal, the issue of these Proceedings.

[5] J. R. Dorfman, An introduction to chaos in nonequilibrium statistical mechanics, Cambridge Lecture Notes in Physics, Cambridge University Press, Cambridge (1999).

[6] P. Allegrini, V. Benci, P. Grigolini, P. Hamilton, M. Ignaccolo, G. Menconi, L. Palatella, G. Raffaelli, N. Scafetta, M. Virgilio, J. Yang, Chaos, Solitons and Fractals 15, 517 (2003).

[7] N. Scafetta, P. Hamilton, P. Grigolini , Fractals, 9, 193 (2001).

[8] P. Grigolini, L. Palatella, G. Raffaelli, Fractals, 9, 439 (2001).

[9] B.B. Mandelbrot, Fractal Geometry of Nature, Freeman, S. Francisco (1988).

[10] P. Allegrini, J. Bellazzini, G. Bramanti, M. Ignaccolo, P. Grigolini, and J. Yang, Phys. Rev. E . 66, 015101 (1-4) R (2002).

[11] Actually, even in the case where the time series under study is random, it takes the diffusion process a finite time to reach the conventional scaling regime. This is so short, however, as to fulfill for moderately large values of $\lambda$ the condition that $C_{j}(\lambda)$ vanishes if the local fluctuations are random. 